\title{
A Qualitative Analysis of Factors Related to Unsafe Work Behaviors Among Environmental Service Workers in the COVID-19 Era: Perspectives of Workers, and Safety Managers: The Case of Government Hospitals in Addis Ababa, Ethiopia.
}

Aiggan Tamene ( $\square$ apublic22@gmail.com )

Wachemo University

Research article

Keywords: Safe work behavior, Environmental service, Occupational health, Coronavirus

Posted Date: October 28th, 2020

DOI: https://doi.org/10.21203/rs.3.rs-96237/v1

License: (c) (i) This work is licensed under a Creative Commons Attribution 4.0 International License. Read Full License 


\section{Abstract}

Background: The Environmental service profession is hazardous, partially because of the work's inherently dangerous nature. Thus, injuries, accidents, and illnesses result in substantial financial and social losses. The most common immediate cause of these work-related incidents is unsafe workbehavior; the first step in taking steps to prevent unsafe behavior is to recognize the factors promoting it. The goal of this study was to (a) investigate the attitudes and perceptions of safety among the employees and safety managers of Coronavirus treatment hospitals in Addis Ababa, Ethiopia and (b) identify the factors that inhibit safe work behaviors.

Methods: Two qualitative data collection approaches, namely key informant interviews and individual indepth interviews, were used to collect data for this study. Twenty-five participants were recruited from three Coronavirus treatment facilities using a modified convenience sampling strategy, and interviews were conducted to gain a detailed understanding of factors that serve as barriers to safe work behavior. The interviews were recorded and transcribed in Amharic (the local language) and then translated into English. Open Code 4.02 was used for thematic analysis.

Results: Poor safety management and supervision, unsafe work environment, and perceptions, skills, and training level of employees were established as the major factors associated with the prevailing unsafe work behavior among environmental service employees.

Conclusions: The present study showed that different types of personal and environmental factors may discourage safe work behavior among environmental service workers. To minimize or remove these risk factors for unsafe behaviors, the individual's obligation is important, but the role of management is critical in providing resources for safe work behavior.

\section{Introduction}

Environmental Services (EVS), is a term used specifically to describe cleaning in health care settings [1]. EVS technicians play a vitally important role in health care. Working with hospital staff to keep patients and staff members safe with proper medical cleaning and disinfection [2]. EVS is so important that it is often referred to as "the first defense against infection control"[3].

Environmental service providers help stop the spread of communicable diseases by regularly cleaning the rooms for patients, nursing units, surgical areas, offices, laboratories, waiting areas, and toilet facilities. They clean furniture, polish floors, as well as empty the trash [4]. When a patient is discharged from the hospital, to prepare for the next patient, it is their job to clean and sanitize every surface with disinfectant. EVS techs make sure patients feel safe at critical times [3]. This is a very important activity in a health care institution. Since a flawed environmental service impacts a hospital's operation and influences the qualities of health care. The proper operation of these services makes way for the operating theatres, inpatient hospitals, intensive care units (ICUs), out-patient centers, etc to function effectively. 
Thus, environmental service workers are very critical in the prevention of hospital-acquired infections [3]. And amid a pandemic characterized by the need for cleanliness- it makes sense that the people cleaning the very hospitals where victims of Coronavirus (COVID-19) fight for their survival are of utmost value. Yet in many hospitals, despite their significance, there is little concern for the safety and health of these staff [5]. Service workers are often the very last to learn about new hospital protocols - as may perhaps have been the case with Coronavirus.

The department of environmental services is hazardous in any hospital, partially due to the inherently unsafe nature of the work done, and accidents often result in substantial losses that impair not only the quality and efficiency of the services rendered by a hospital but also endanger the life and livelihood of workers. These employees face numerous risks such as physical (excessive temperature, humidity, physical stress), chemical (contact cleaning of goods and disinfection, chemotherapeutic disposal), ergonomic (excessive physical exertion, repeatability of activity, poor posture), cognitive ergonomics (social devaluation of jobs, loss of motivation), and biological (contact with cutting and piercing material during transport to box disposal) that favors the development of certain diseases: such as musculoskeletal disorders, contact with dermatitis, low back pain and infectious diseases [3,5-6].

Most of these accidents and injuries emerging from workplaces are attributed to workers' unsafe behaviors, which are also an indication of system deficiency and hazardous work-environment [7]. Add a virus that is transmitted primarily between humans through respiratory droplets and contact routes to the mix and the situation becomes significantly riskier.

Many countries have encountered lots of challenges in clinical settings when responding to COVID-19. Experience has shown that the health care workforce $(\mathrm{HCW})$ is one of the most vulnerable groups to COVID-19 infection, especially of a nosocomial nature [8]. Hospital workers in departments considered lower risk, like cleaners, sweepers, and others, are more at risk of contracting the virus than doctors working in intensive care units, according to a recent study of several British hospitals [9]. Researchers noted this could be because doctors working in the ICU take all the precautionary steps when treating patients with Coronavirus [9].

On 13 March 2020, Ethiopia, the second-most populous nation in Africa, with 112 million people, confirmed its first case of COVID-19. Confirmed cases of COVID-19 have since surpassed 68,000 . A total of 40,000 people are currently undergoing treatment, of which close to 300 are in serious medical condition. Nearly all confirmed cases are confined to urban areas $(77 \%)$, with most of the cases $(67 \%)$ occurring in the capital, Addis Ababa [10]. This figure has made Ethiopia the leading nation in East Africa in the number of confirmed cases and the ninth leading African country in confirmed cases as well as deaths.

Currently, there are 11 government-owned hospitals in Addis Ababa out of which 9 operate as treatment centers for COVID-19. Despite numerous studies on safe work behaviors among front-line health care workers, however, there is limited information on factors associated with unsafe work behaviors among environmental service workers in the COID-19 era. It is against this background that, this article aims to 
examine the attitudes and expectations of environmental service employees and safety managers in Addis Ababa government hospitals in the COVID-19 era and describe the factors that hinder safe work behaviors among these workers in 2020 .

\section{Methods And Materials}

The study was conducted in three government hospitals in Addis Ababa city, the Ethiopian capital, from June 25 - July 22, 2020. The three government hospitals were purposively selected for this study as a result of their large COVID-19 patient flow and the high demand for efficiency prevalent in their environmental services divisions.

The study team consisted of one main researcher, four professional data collectors in the field of health and safety, and one supervisor. The supervisor and the four data gatherers were given two days-long intensive training. During the training, lessons on data collection techniques and how best to handle the study participants were discussed in detail

The study had two sets of partakers. One group consisted of personnel from the environmental services divisions, and in-hospital Infection Control and Patient safety officers were members of the other group. These officers are environmental and occupational health practitioners in charge of supplying hospital employees with personal protective equipment. They are also responsible for overseeing the cleaning efforts. An on-site survey was carried out at all hospitals to single out the study participants. In total, 69 employees were identified as working in the environmental service departments. Infection control and patient safety departments had 15 professionals.

The study's design is qualitative. Semi-structured key informant interviews (KII) and in-depth interview guides (IDI) were the data-gathering instruments used in this research. After a detailed review of the related literature, the research team developed the guides used in this study (See Additional file 1). The data collection guides revolved around the perceptions and safety experiences of the participants in the workplace in the COVID-19 era and their perceived impediments to a safe place of employment. The guide also had sections related to the daily life of the workers in the hospitals and workplace relationships.

The interview guides used with both groups of participants in the study were made similar to ensure comparability of the responses they obtained. To select participants, this study used an updated, purposive sampling. A list of environmental service personnel was provided to the researcher that randomly selected workers from the list and invited people to participate. Furthermore, employees who had accidents, injuries, or work-related illnesses or witnessed accidents, injuries, or work-related illnesses of colleagues in the recent past were invited to participate. In addition to the in-depth interviews, key informant interviews were also conducted at the selected hospitals. Inclusion in the KIls was determined based on the convenience of the participants in the study and willingness to participate. 
The data collection schedule was set with both hospital managers and the workers themselves. When the data collection schedule was set, an agreement was also reached with hospital managers and the workers to hold the interviews in separate offices to ensure the privacy of the participants. Ensuring the privacy of participants was needed to encourage the participants' genuine involvement and share as sources of the data needed for the study. Before they agreed and signed informed consent forms, the goal of the study was clearly explained to the participants. Also, the participants were given confirmation of the research team's commitment to maintain confidentiality and keep the exact sources of the opinions raised in the interview sessions anonymous.

The interviews lasted about 35-45 minutes; when the answers were unclear or ambiguous, or to obtain more detailed or in-depth information, probing questions were used. Just one interview was conducted in a day and most of the data were transcribed on the same day. This made it possible to raise some salient points that needed more focus in the subsequent sessions with the other participants. Both the key informant interviews and in-depth interviews were held until the data reached saturation - a point where repeated trends were visible in the narratives of the participants. A total of 25 interviews were conducted before saturation was reached. In addition to the participants in the study, the interview consisted of an interviewer, a note-taker, and an observer. Physical distancing was preserved during the data collection.

For data analysis, Open code 4.03 was used, which followed a thematic framework. The information gathered was transcribed verbatim in the local language (i.e., Amharic). The data was later translated into English by the same person. Following the translation, the original transcript was analyzed with its translated version. This meant that the translated objects lacked any inconsistencies in words, definitions, and contents. Next, data were validated and themes were established in an inductive and deductive method based on the issues that arose from the interviews with the study participants. Also, index cards were used to develop codes, which were then applied systematically to the themes. This process ensured the theme mapping and facilitated the application of the codes and themes in question. Finally, a list of codes and themes was extracted and applied to the data. Related verbatim quotes are used to help in the interpretation of the data when presenting the data.

Ethical clearance was obtained from Wachemo University College of Medicine and Health Sciences' institutional review board (IRB). A letter of permission had been obtained from the Addis Ababa City Health Bureau before data collection. Also, respondents' participation was based on their full acceptance and consent.

\section{Result}

Workers in the departments of the environmental services were generally grouped into smaller units that were specialized in various tasks. Different departmental activities are designated for the units. For example, the cleaning of patient rooms, the cleaning of nursing units, the cleaning of surgical areas, the cleaning of administrative offices, the cleaning of laboratories, the cleaning of waiting areas, the cleaning of toilet facilities, the cleaning of hospital premises, and the collection and emptying of garbage from all 
of the above-listed sites are among the duties of the environmental service divisions of all three hospitals. 25 Hospital staff took part in this study in total. Of these, 19 were environmental service staff, and six were employees from the Infection Control and Patient Safety Departments. 20 of the study participants (i.e. $80.0 \%$ ) were female. The mean age of the participants was 38.2 with Standard deviation (SD) \pm 8.2 and range $18-59$.

\section{Factors Related to Unsafe Work Behaviors}

\section{Themes and sub-themes (categories) identified}

Three main themes emerged from the participants' perspectives concerning the factors associated with unsafe work behaviors- (1) Poor management and supervision of safety, (2) Unsafe workplace conditions, (3) Perceptions, skills, and training level of workers (Table 1).

Table 1 Classification of themes, categories, and codes according to the thematic analysis 


\begin{tabular}{|c|c|c|}
\hline Main themes & Subthemes (Category) & Sub-categories \\
\hline \multirow{7}{*}{$\begin{array}{l}\text { Poor } \\
\text { management } \\
\text { and } \\
\text { supervision } \\
\text { of safety }\end{array}$} & \multirow[t]{4}{*}{$\begin{array}{l}\text { Ineffective safety } \\
\text { management }\end{array}$} & $\begin{array}{l}\text { - Lack of ready access to personal protective } \\
\text { equipment (PPE) in the workplace. }\end{array}$ \\
\hline & & Poor design \& quality of available PPE \\
\hline & & - $\quad$ Insufficient training \\
\hline & & Workplace policy gaps \\
\hline & \multirow{3}{*}{$\begin{array}{l}\text { Poor monitoring and } \\
\text { supervision of safety }\end{array}$} & Negligent control of PPE compliance \\
\hline & & Haphazard safety inspections \\
\hline & & Haphazard inventory tracking \\
\hline \multirow{8}{*}{$\begin{array}{l}\text { Unsafe } \\
\text { workplace } \\
\text { conditions }\end{array}$} & \multirow{7}{*}{$\begin{array}{l}\text { Unsafe psychological } \\
\text { environment }\end{array}$} & Fatigue relating to work \\
\hline & & - $\quad$ Excessive loads of work \\
\hline & & $\begin{array}{l}\text { Delayed payments for wages and salaries that } \\
\text { minimize security incentives }\end{array}$ \\
\hline & & $\begin{array}{l}\text { - Condescending safety supervision and } \\
\text { maltreatment }\end{array}$ \\
\hline & & - $\quad$ Stress related to work \\
\hline & & Separation from family \\
\hline & & Low organizational dedication to safety \\
\hline & $\begin{array}{l}\text { Unsafe Physical } \\
\text { environment }\end{array}$ & $\begin{array}{l}\text { Impaired concentration due to a fast-paced } \\
\text { environment, excessive noises, and increased traffic }\end{array}$ \\
\hline \multirow{4}{*}{$\begin{array}{l}\text { Perceptions, } \\
\text { skills and } \\
\text { training level } \\
\text { of workers }\end{array}$} & \multirow[t]{3}{*}{ Workers' perceptions } & Concerns of PPE interfering with work \\
\hline & & - Hazards that are 'normalized' overtime \\
\hline & & $\begin{array}{l}\text { Human factor-related phenomena such as culture, } \\
\text { beliefs, and attitudes }\end{array}$ \\
\hline & $\begin{array}{l}\text { Lacking skills to } \\
\text { manage hazards }\end{array}$ & $\begin{array}{l}\quad \text { Lack of experience and skills in dealing with } \\
\text { hazards }\end{array}$ \\
\hline
\end{tabular}

\section{Theme one: Poor management and supervision of safety.}

\section{Ineffective safety management}

Almost all of the participants described a general lack of PPE as a major barrier to safe behaviors. There was also evidence of a tendency to imply that hospital administrators have failed to understand the role that timely, adequately, and appropriately provided PPE can play in the control of hazards. As stated 
below, one respondent, for example, expressed frustration with the shortage of critical PPE supplies in the hospital. (P12 refers to participant 12)

P12: One barrier to safe behaviors is that the personal protective equipment if available is very worn-out and defective. I've been working here for 6 months, and so far I haven't gotten any PPE. I use the worn-out equipment that was used by those who worked here before the pandemic.

Moreover, participants indicated that a lot of personal protective equipment such as masks, gloves, and goggles were either too large or too small for them to comfortably function. So it seems the workers didn't have anything to use.

P4: Although I know we are supposed to use facemasks while cleaning, I prefer instead to cover my face with a scarf because the masks the hospital gives us do not fit properly.

Key informants considered in this study had their administrative views regarding the gaps in the supply of PPE in the departments of environmental services (K2 refers to Key-informant 2)

K2: ... We [hospitals] survive with our existing old gear because the pandemic has strained all available resources. We take what we can get and use it to the best of our advantage; the government provides what it has and what it can.

The matter of training was raised by the respondents as another important reason for the failure of the staff to fully exercise safe work behavior. Participants addressed their training and shared their frustration with the training frequency (and adequacy by implication).

P16: They do train us but in my view, it's not adequate. It was only given to us once since the pandemic began ... l'd have preferred it if it were at least once every few weeks. A lot of new employees have come and gone after the training. Thus, beginners are usually left to their own devices to find out why the equipment is needed and how and when it is used.

Key informants clarified that given the costs that go into scheduling a training more than once a year; priority was given to clinical workers. That is illustrated by the following extract.

K2: Training on how to protect themselves from COVID-19, as well as steps for appropriate PPE use, is given to all staff. The training was given to physicians and nurses twice this year, but it was given once to the other staff members. We must prioritize our resources, and use them more where they are most needed

The lack of best practices in safety management was also visible from the participants' testimonies. This impacted the work behavior of the staff. For instance, a worker's state of employment, i.e. whether one is a permanent or temporary employee, has a bearing on their access to the training or PPE, though both types of employees might have the same opportunity of exposure. 
P13: as you know, safety goggles protect us from exposure to droplets from infected individuals, but it's hard for those of us working under temporary contracts since our bosses say it's only for permanent employees and not for others.

P8: Permanent hospital employees were given a participation fee of \$7 (300 Ethiopian birr) for COVID-19 prevention training, but for workers employed under contractual terms there was no such fee. So many of our colleagues skipped the training out of anger

\section{Poor monitoring and supervision of safety}

Maintaining workplace safety and health in any workplace is as much the duty of the manager as it is the responsibility of the employees themselves. In the present study, participants discussed in detail the lack of strict supervision and scheduled inspection as one of the major barriers to safe work behavior among the cleaning staff.

P5: Supervisors came this morning but they asked us how the work was going and not about our protection. ... There's no concern about what we're missing, more so when we're on the night shift.

In addition to these weak supervisions regimes, the lack of successful inventory management systems inside the hospitals was recognized as a factor that greatly impedes the pursuit of healthy work behaviors by employees.

P8: Often the hospital wards get so loaded with COVID patients that we have to work overtime and when we ask for mask or glove replacements, our superiors say okay, but once we enter the storeroom, the item is out of stock. We can't afford to lose our jobs so we work under these circumstances.

\section{Theme two: Unsafe workplace conditions}

\section{Unsafe psychological environment}

The data collected in the present study shows the prevalence of stressful workplace conditions. For example, many participants described departmental workload as stressful due to demands for productivity. They also mentioned the sacrifices that they had to make between job speed and workplace safety.

P16: We feel the pressure. We feel that any interruption in our services could cause severe problems. These patients may be individuals who we know or whom we work with. We see them fight for their lives and feel like we must make their stay here as comfortable as possible. So it's hard when you're feeling that, that constant urge to fix everything.

Key informants did seem to agree with some aspects of the workers' characterization of the workplace conditions 
K4: Any worker who develops or is suspected of developing symptoms gets placed in our isolation centers. So the alienation from your family and the concerns of your job security is constantly at the back of your mind ... you suffer psychologically, of course, ideally not to the point of stress-induced accidents

Others felt that they were working under extreme pressure. They stated that some mid-level staffs seem to believe, perhaps incorrectly, that exhibiting an authoritarian attitude towards workers is the way to enhance and maximize productivity and achieve objectives in the workplace.

P2: Often, because of personal or family issues, people may miss work, so our bosses come here to check who is absent and late, not to ask us if they can do something for us... Most of the questions that are asked here are questions such as has the floor been cleaned? Have the toilets been washed? And if not; they go on a long rant but never really ask why

The apparent gap between the anticipation of the participants to benefit from reward programs and hospital administrators' inability to set up effective programs seems to affect the excitement of the employees for safe work behaviors.

P1: Here those who work hard are not valued; in other divisions, I have, many acquaintances, and for their selfless duties they get bonuses and rewards, this should be introduced here to encourage safety, to endorse hard work.

\section{Unsafe Physical environment}

Noise, increased traffic, and other changeable environmental variables impacting workplace safe behaviors were also discussed.

K6: These are unusual times, there are people in the halls, in-patient wards are packed ... people here are crying for relatives ... people there are gasping for air ... the staff is constantly interacting with each other... your voice is being muffled with masks on ... so you have to repeat things to your colleagues or you are momentarily pulling them down to be audible... COVID-19 has made hospitals congested beyond belief.

\section{Theme three: Perceptions, skills, and training level of workers}

\section{Workers' perceptions}

Human factor-related phenomena such as culture, beliefs, and attitudes too became apparent as a major impediment to safe work behaviors. A repeated allusion from the interviewees was that some had a negative attitude towards safe work behaviors within the workforce.

P18: Several workers in our hospital believe that using personal protective equipment is a waste of time and that using masks increases the incidence of syncope because it doesn't give the brain enough oxygen, particularly the older employees. They say to us the only security I need is God. 
For some, perceptions of minimal health threat from the Coronavirus dissuaded safe work behaviors.

P12: Whether or not you take precautions, we're all bound to inevitably get it ... I hear most people don't even notice when they get it ... it's like the flu at best

And yet, for a few others, age-old traditions such as herbal remedies provided them with a perceived safety net.

P6: ... There is a reason why for centuries the "feto" plant (Lepidium sativum) has been celebrated as a miracle for any respiratory disease... I know people who have recovered from the Coronavirus using just that.

Similarly, subgroup demographic characteristics (e.g., job position and experience level) also had a tremendous impact on safety perceptions. For some, the key factor in avoiding infections was to rely on experience and self-confidence while doing the work; this is reflected in their optimistic views of themselves and their conceptualizations of advantageous improvements related to age, such as the ability to execute tasks with minimal risk to oneself.

P10: the techniques of working safely and preventing any kind of danger have been perfected by seasoned staff, so I feel like I can function perfectly well if there is a pandemic or an outbreak. Guidelines add little to my know-how

Other young participants seem to have accepted that more seasoned employees are less likely than their novice peers to get a work-related illness. One worker related this to the levels of promotion that, as seen in the excerpt below, come with experience.

P6: Older and more seasoned staffs are more likely to be in team leader roles and are often active in activities that do not require them to face dangerous conditions.

\section{Lacking skills to manage hazards}

In some cases, participants were quick to point out that many of their colleagues, especially the new ones, lacked the basic skills and expertise needed for employees to properly perform their particular tasks. In their view, this led to many employees applying untested work procedures and normalizing risks in the workplace.

P14: We see inexperienced workers take greater risks when conducting routine tasks. The hospital administration frequently assigns the task of training to employees who have been on the job longer. But we don't get additional time to do that many times; we still need to finish our jobs. Therefore we leave it to the new employee to ask questions. So if you're inexperienced; cleaning hospitals in the COVID-19 era it shouldn't be your meal ticket.

\section{Discussion}


The purpose of this study was to investigate the perceptions of safety at work in three COVID-19 treatment hospitals in Addis Ababa, Ethiopia, by environmental service workers and safety managers, and to identify factors that inhibit safe work behaviors. Using KIIs and IDIs, the qualitative data gathered helped to shed light on some important factors contributing to unsafe work behaviors among these workers. The results are important to researchers, safety experts, and administrators since they sometimes ignore possible hazards in the forgotten pandemic heroes' workplaces.

The Coronavirus pandemic has put pressure on the health care system to prepare health care workers for increased patient care. It is understood that performing effectively in any healthcare setting requires the availability of appropriate facilities and adequate equipment and supplies [11]. In the present study, the lack of sufficient and appropriate personal protective equipment in hospitals was established as a major obstacle to the practice of safe work behaviors by employees. This is similar to the findings of other earlier studies on PPE use among health care workers, where staffs have been found to decrease the use of PPE if they find it unacceptable, inefficient, or impeding vision [12-13]. Health workers should be equipped with PPE of an acceptable quality standard for effective use. This relates to equipment capable of reducing physiological stresses, facilitating engagement, and maintaining comfort. Otherwise, staff loyalty to PPE will be jeopardized [14].

Similarly, incorrect and insufficient safety training is correlated with the vast majority of incidents in a health care environment [15]. Any behavior in a health-related workplace must be sustained by a high degree of knowledge and empirical evidence [16]. In a pandemic, this is particularly true. The lack of appropriate and up-to-date safety training in the study area was seen as contributing to the inability of the workers to maintain safety protocols effectively in the workplace. Participants at various times raised questions about the appropriateness of the workplace training they are offered. They said that the training given only once since the beginning of the pandemic is much less than what is required to make it possible for them to function effectively. Hospital training should draw from a range of health care occupations since staffs in the environmental service departments are far more vulnerable to COVID-19 than intensive care unit doctors [9]. After all, workers in the EVS departments tend not to have adequate formal education in health care.

Hospital policy as a feature may affect the observance of safety protocols [17]. Increasingly, efforts to implement best practice guidelines have continually been underlined as a core component of occupational safety. Best practices have been described by the national institute of occupational safety and health (NIOSH) as a collection of critical elements that prioritize a hazard-free work atmosphere and acknowledge the important role of job-related factors in the health and well-being of workers [18]. Deficiencies in the execution of best practice policies will contribute to lower rates of utilization and compliance of safety measures. This exposes employees ' health to work-related illness [19]. This was also true in the current study.

The overriding need for a safe environment to prevail at the place of work was an issue of an in-depth conversation throughout the interviews. For example, routine audits of equipment stock emerged as an 
indicator of a favorable workplace safety environment during the interviews. Another indication of the safety environment was regular supervision of workplace conditions. This finding is in line with the results of several other studies that indicated that improving safety management systems may be a great tool for improving unsafe work behaviors [20-22].

Another noteworthy finding relates to unsafe conditions in the workplace. Workers' feelings about the hospital administrative models were seen as acting as obstacles to their workplace safety behaviors. The workers had the feeling that the hospitals' administrative models were designed to ensure a technical focus on outcomes before people and their environment. This is representative of the social environment's effect on safe work behaviors. Indeed, poor social working environments may function as external stressors and have been linked to safety perceptions. The current findings provide added support for previous studies that stress the importance of safe workplace environments. It has been stated previously that factors in the work environment, such as resource levels, communication among employees, and the working system, have the greatest impact on the sense of belonging of staff to the organization and lead to positive safety behaviors [14, 23].

Likewise, the physical environment as an aspect of the workplace environment directly affects the human sense and can slow interpersonal interactions and therefore productivity [24]. In the present study, participants complained that the unfamiliar working environments produced by the pandemic including noise and elevated crowds hindered their focus. They perceived these conditions as detrimental to working safely. Noise has previously been reported to have a detrimental effect on communication, increasing levels of dissatisfaction while decreasing efficiency. A reason for this is that as noise levels increase, communication becomes progressively more difficult [24]. Similarly, a health worker's ability to work effectively in a crowd of numerous uncontrolled patients is restricted [24]. Thus, in the COVID-19 period, today's management has to adjust the style of working to bring about successful improvements in these aspects. Management must invest more time monitoring these work environment conditions, rather than micromanage.

In terms of perceptions, skills, and training of staff, in many situations, doing routine tasks and duties was found to have led to a false sense of invulnerability, subsequent non-compliance, and increased risktaking. Empirical studies provide evidence that, when performing routine tasks, employees can feel that they are not at risk [25]. Similarly, some participants displayed misconceptions regarding PPE interference with productivity or perceptions of a marginal threat to health from the Coronavirus. These kinds of perceptions inevitably contribute to higher risk-taking and unpreparedness for the next unknown. Via scientific problem-based training programs, however, these beliefs, behaviors, and knowledge gaps can be modified [26].

There are some limitations to the current study. This is a three-site study and the results in their entirety are not likely to be indicative of other hospitals. Also, participant responses could be skewed to provide sociably desired responses as a result of social desirability. This means that it is difficult to eliminate opportunities for hesitation among certain participants to represent their true experiences. In addition, the 
findings in the present study are based on the perceptions, rather than hard evidence, of the study participants. Recorded findings have not been independently confirmed. While perceptions are important, they can often be tainted by excitement, vested interests, and thus fail to reflect real circumstances accurately as they occur.

In the future, although the Coronavirus (COVID-19) pandemic, along with very difficult work environment, and stretched healthcare systems, might make it difficult to collect data on the number of EVS staffs who drop out due to infectious diseases that may result from unsafe work behavior, efforts to collect such data should be undertaken whenever possible. Similarly, to help educate administrators and policymakers, an evaluation of the costs associated with unsafe work behavior-related illnesses and workers and process downtime as a result of those illnesses should be conducted. Lastly, the current study showed that training is a significant determinant of safe work behaviors; it was, however, analyzed in a general way. Future research will need to further disaggregate it and study the form and pace of training, the material offered, and the impact of previous experience of the staff on this training.

\section{Conclusion}

The present study showed that different types of personal and environmental factors may discourage safe work behavior among environmental service workers. To minimize or remove these risk factors for unsafe behaviors, the individual's obligation is important, but the role of management is critical in providing resources for safe work behavior. The resources include PPE, proper training, and a supportive work environment. In conclusion, the current research offers a systematic and practical evaluation of obstacles to healthy work behaviors through the incorporation of employees' experiences and infection prevention officers. Previous researches were performed using quantitative approaches to assess the main factors influencing safety in healthcare settings while often missing the perceptions of the staff. These perspectives provide an essential context for overcoming the obstacles staffs face to safely perform their duties.

\section{List Of Abbreviations}

Coronavirus (COVID-19), Environmental Services (EVS), Health care workforce (HCW), Institutional review board (IRB), In-depth interview (IDI), Key informant interview (KII), National Institute of occupational safety and health (NIOSH), Personal protective equipment (PPE), Standard deviation (SD)

\section{Declarations}

\section{Ethics approval and consent to participate}

Ethical clearance and approval were received from the Ethical Review Committee at Wachemo University. The requisite support letter was obtained from the Health Bureau of Addis Ababa. Permission from all hospital medical directors was obtained before the study. Written consent for the research was given by 
all participants. All participants were properly advised of the intent of the study and were notified of their right to not participate in the study or to stop the interview. Furthermore, all data acquired in the study was kept confidential and safe.

\section{Consent for publication}

Not applicable

\section{Availability of data and materials}

The qualitative datasets that were generated and analyzed during this research are not publicly accessible and because of participant confidentiality, cannot be shared, as the findings are going to be submitted to the Addis Ababa City Health Bureau and all COVID-19 treatment centers in the study area, participants may be potentially identifiable from the information contained in the data. De-identified transcripts of the interviews, however, may be available on reasonable request from the corresponding author.

\section{Competing interests}

The author declares that he has no competing interests

\section{Funding}

The author received no financial support for the research, authorship, and/or publication of this article.

\section{Author's Contributions}

AT was involved in the conceptualization and investigation, study design, overall supervision, analysis, writing, and approval of the final manuscript.

\section{Acknowledgments}

The author would like to thank the University of Wachemo, the Addis Ababa Health Bureau, and the medical directors of the selected hospitals. Special thanks to the staff for their time and patience and the data collectors and supervisors for their rigorous hard work.

\section{Author's Information}

${ }^{1}$ Department of Public Health, College of Medicine and Health Sciences, Wachemo University, P.O.BOX:667, Hosaena, Ethiopia.

\section{References}

1. Leas B, Sullivan N, Han J, Pegues D, Kaczmarek J, Umscheid C. Environmental Cleaning for the Prevention of Healthcare-Associated Infections2015. 
2. Carling P, Parry M, Rupp M, Po J, Dick B, Beheren S. Improving Cleaning of the Environment Surrounding Patients in 36 Acute Care Hospitals. Infection control and hospital epidemiology: the official journal of the Society of Hospital Epidemiologists of America. 2008;29:1035-41.

3. Dancer S. The role of hospital cleaning in the control of hospital-acquired infection. The Journal of hospital infection. 2009;73:378-85.

4. Peters A, Otter J, Moldovan A, Parneix P, Voss A, Pittet D. Keeping hospitals clean and safe without breaking the bank; summary of the Healthcare Cleaning Forum 2018. Antimicrobial Resistance \& Infection Control. 2018;7.

5. Ilesanmi O, Omotoso B, Falana D. Hazards of hospital cleaners in a tertiary health facility in Southwest Nigeria. International Journal of Occupational Safety and Health. 2015;4.

6. Faremi F, Olatubi M, Ogunlade B, Ajayi O. Knowledge of Occupational Hazards among Cleaning Workers: A Study of Cleaners of a Nigerian University Knowledge of Occupational Hazards among Cleaning Workers: A Study of Cleaners of a Nigerian University. 2018.

7. Dodoo JE, Al-Samarraie H. Factors leading to unsafe behavior in the twenty-first-century workplace: a review. Management Review Quarterly. 2019 2019/11/01;69(4):391-414.

8. Eleojo I, Enitan S, Enitan C. COVID-19 among Healthcare Workers: Risk of Exposure, Impacts, and Biosafety Measures -A Review. 2020:534-48.

9. Shields A, Faustini SE, Perez-Toledo M, Jossi S, Aldera E, Allen JD, et al. SARS-CoV-2 seroprevalence, and asymptomatic viral carriage in healthcare workers: a cross-sectional study. Thorax. 2020:thoraxjnl-2020-215414.

10. Shigute Z, Mebratie AD, Alemu G, Bedi A. Containing the spread of COVID-19 in Ethiopia. J Glob Health. 2020 Jun;10(1):010369.

11. Salehi H, Pennathur PR, Da Silva JP, Herwaldt LA. Examining health care personal protective equipment use through a human factors engineering and product design lens. American journal of infection control. 2019 May;47(5):595-8.

12. Fischer WA, 2nd, Weber D, Wohl DA. Personal Protective Equipment: Protecting Health Care Providers in an Ebola Outbreak. Clinical therapeutics. 2015;37(11):2402-10.

13. Baloh J, Reisinger H, Dukes K, Silva J, Salehi H, Ward M, et al. Healthcare Workers' Strategies for Doffing Personal Protective Equipment. Clinical infectious diseases: an official publication of the Infectious Diseases Society of America. 2019;69:S192-S8.

14. Den Boon S, Vallenas C, Ferri M, Norris S. Incorporating health workers' perspectives into a WHO guideline on personal protective equipment developed during an Ebola virus disease outbreak. F1000Research. 2018;7:45.

15. Shehu Mashi M, Subramaniam C, Johanim J. The Effect of Safety Training and Workers' Involvement on Healthcare Workers Safety Behavior: The Moderating Role of Consideration of Future Safety Consequences. International Journal of Business Management (IJBM). 2016;1:2016-62.

16. Sorensen G, Sparer E, Williams JAR, Gundersen D, Boden LI, Dennerlein JT, et al. Measuring Best Practices for Workplace Safety, Health, and Well-Being: The Workplace Integrated Safety and Health 
Assessment. Journal of occupational and environmental medicine. 2018;60(5):430-9.

17. Little J, Coughlan B. Optimal inventory policy within hospital space constraints. Health care management science. 2008;11:177-83.

18. Steege A, Boiano J, Sweeney M. NIOSH Health and Safety Practices Survey of Healthcare Workers: Training and Awareness of Employer Safety Procedures. American journal of industrial medicine. 2014;57.

19. Sawada SI, Kuklane K, Wakatsuki K, Morikawa H. New development of research on personal protective equipment (PPE) for occupational safety and health. Industrial health. 2017;55(6):471-2.

20. Bakhsh K, Ahmad N, Tabasum S, Hassan S, Hassan I. Health hazards and adoption of personal protective equipment during cotton harvesting in Pakistan. The Science of the total environment. 2017 Nov 15;598:1058-64.

21. Balkhyour MA, Ahmad I, Rehan M. Assessment of personal protective equipment use and occupational exposures in small industries in Jeddah: Health implications for workers. Saudi journal of biological sciences. 2019 May;26(4):653-9.

22. Kang J, O'Donnell JM, Colaianne B, Bircher N, Ren D, Smith KJ. Use of personal protective equipment among health care personnel: Results of clinical observations and simulations. American journal of infection control. 2017 Jan 1;45(1):17-23.

23. von Vultée P. Healthy work environment - A challenge? International journal of health care quality assurance. 2015;28:660-6.

24. Mj E, Eu A, Nm P. Impact of Workplace Environment on Health Workers. Occupational Medicine \& Health Affairs. 2017;05.

25. Nordlöf H, Wiitavaara B, Winblad U, Wijk K, Westerling R. Safety culture and reasons for risk-taking at a large steel-manufacturing company: Investigating the worker perspective. Safety Science. 2015;73:126-35.

26. John A, Tomas M, Cadnum J, Mana T, Jencson A, Shaikh A, et al. Are Healthcare Personnel Trained in Correct Use of Personal Protective Equipment? Open Forum Infectious Diseases. 2015;2.

\section{Supplementary Files}

This is a list of supplementary files associated with this preprint. Click to download.

- AdditionalFile1.docx 